\title{
3D Structure Prediction of TAS2R38 Bitter Receptors Bound to Agonists Phenylthiocarbamide (PTC) and 6-n-Propylthiouracil (PROP)
}

\author{
Jun Tan, ${ }^{\dagger, \dagger}$ Ravinder Abrol, ${ }^{\dagger}$ Bartosz Trzaskowski, ${ }^{\dagger}$ and William A. Goddard, III*, ${ }^{\dagger}$ \\ ${ }^{\dagger}$ Materials and Process Simulation Center (MC-139-74), California Institute of Technology, 1200 E. California Boulevard, Pasadena, \\ California 91125, United States
}

\section{Supporting Information}

\begin{abstract}
The G protein-coupled receptor (GPCR) TAS2R38 is a bitter taste receptor that can respond to bitter compounds such as phenylthiocarbamide (PTC) and 6-n-propylthiouracil (PROP). This receptor was chosen because its four haplotypes (based on three residue site polymorphism) hTAS2R38 $8_{\text {PAV }}$, hTAS2R38 $8_{\text {AVV }}$ hTAS2R38 $8_{\text {AAV }}$ and hTAS2R38 $8_{\mathrm{PVV}}$ are known to have dramatically different responses to PTC and PROP. We aimed to identify the protein-ligand interaction features that determine whether the bitter taste signal from this receptor is sent to the cortex. To do this we predicted the 3D structures of the TAS2R38 bitter taste receptor using our new BiHelix and SuperBiHelix Monte Carlo methods (No experimental determinations of the $3 \mathrm{D}$ structure have been reported for any taste receptors.). We find that residue 262 (2nd position in the polymorphism) is involved in the interhelical hydrogen bond network stabilizing the GPCR structure in tasters (hTAS2R $38_{\text {PAV }}$, hTAS2R38 $8_{\text {AAV }}$ and hTAS2R $38_{\text {PVV }}$ ), while it is not in the nontaster (hTAS2R38 $\left.8_{\text {AVI }}\right)$. This suggests that the

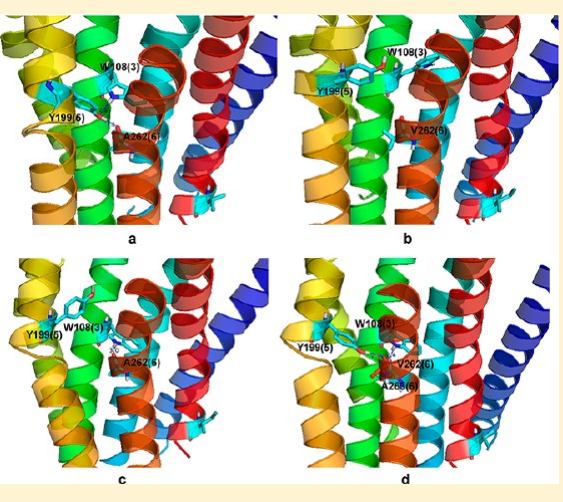
hydrogen bond interactions between TM3 and TM6 or between TM5 and TM6 may play a role in activating this GPCR. To further validate these structures, we used the DarwinDock method to predict the binding sites and 3D structures for PTC and PROP bound to hTAS2R38 ${ }_{\mathrm{PAV}}$, hTAS2R38 $8_{\mathrm{AVI}}$, hTAS2R38 $8_{\mathrm{AAV}}$ and hTAS2R38 $8_{\mathrm{PVV}}$, respectively. Our results show that PTC and PROP can form H-bonds with the backbone of residue 262 in the tasters (hTAS2R38 $8_{\text {PAV }}$ hTAS2R $38_{\mathrm{AAV}}$, and hTAS2R38 $8_{\mathrm{PVV}}$ ) but not in the nontaster (hTAS2R38 $8_{\mathrm{AVI}}$ ). Thus it appears that the hydrogen bond interaction between TM3 and TM6 may activate the receptor to pass the ligand binding signal to intracellular processes and that the H-bond between agonists and residue 262 in tasters is involved in the bitter tasting. This is in agreement with experimental observations, providing validation of the predicted ligand-protein complexes and also a potential activation mechanism for the TAS2R38 receptor.
\end{abstract}

\section{INTRODUCTION}

Bitter taste receptors, denoted as TAS2R, are seven-transmembrane (TM) domain G protein-coupled receptors (GPCRs) that are quite distant from the well-studied class A GPCRs which include adrenocepters (for which X-ray crystal structures are known for turkey $\beta 1$ and human $\beta 2$ ), dopamine receptors (X-ray crystal structure is known for human D3), rhodopsin receptor (X-ray crystal structure is known for bovine), and adenosine receptor (X-ray crystal structure is known for human A2a). It is generally believed that the bitter taste has evolved to warn us not to ingest potentially harmful compounds, ${ }^{1}$ although the correlation between toxicity and bitter taste thresholds of compounds varies depending on the nutritional habits of the animals being studied. $^{2}$

In humans, the bitter taste is mediated by a family of approximately 30 bitter taste receptors (TAS2Rs) expressed in taste receptor cells. ${ }^{3-7}$ Extracellular bitter substances [such as phenylthiocarbamide (PTC) and 6-n-propylthiouracil (PROP)] bind to these receptors, which initiates a downstream cascade of signal transduction within the cell, which ultimately results in bitter taste perception. ${ }^{8,9}$ Interestingly it has been recently determined that TAS2R-type taste receptors are expressed not just in the

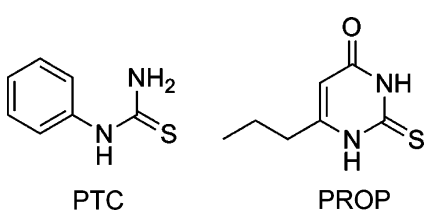

Figure 1. Molecular structures of phenylthiocarbamide (PTC) and 6-npropylthiouracil (PROP).

gustatory system but also in subsets of cells within the mammalian gastrointestinal (GI) tract, where they mediate nutrient assimilation and endocrine responses. Indeed the TAS2R38 agonist PTC activates bitter taste receptors in the GI track to stimulate ghrelin secretion, modulate endogenous ghrelin levels, and alter glucose and insulin homeostasis. ${ }^{10-12}$ This finding suggests that development of agonists for hTAS2R38 can potentially provide lead compounds for treatment of diabetes and obesity. Taste receptors have also

Received: March 12, 2012

Published: June 1, 2012 


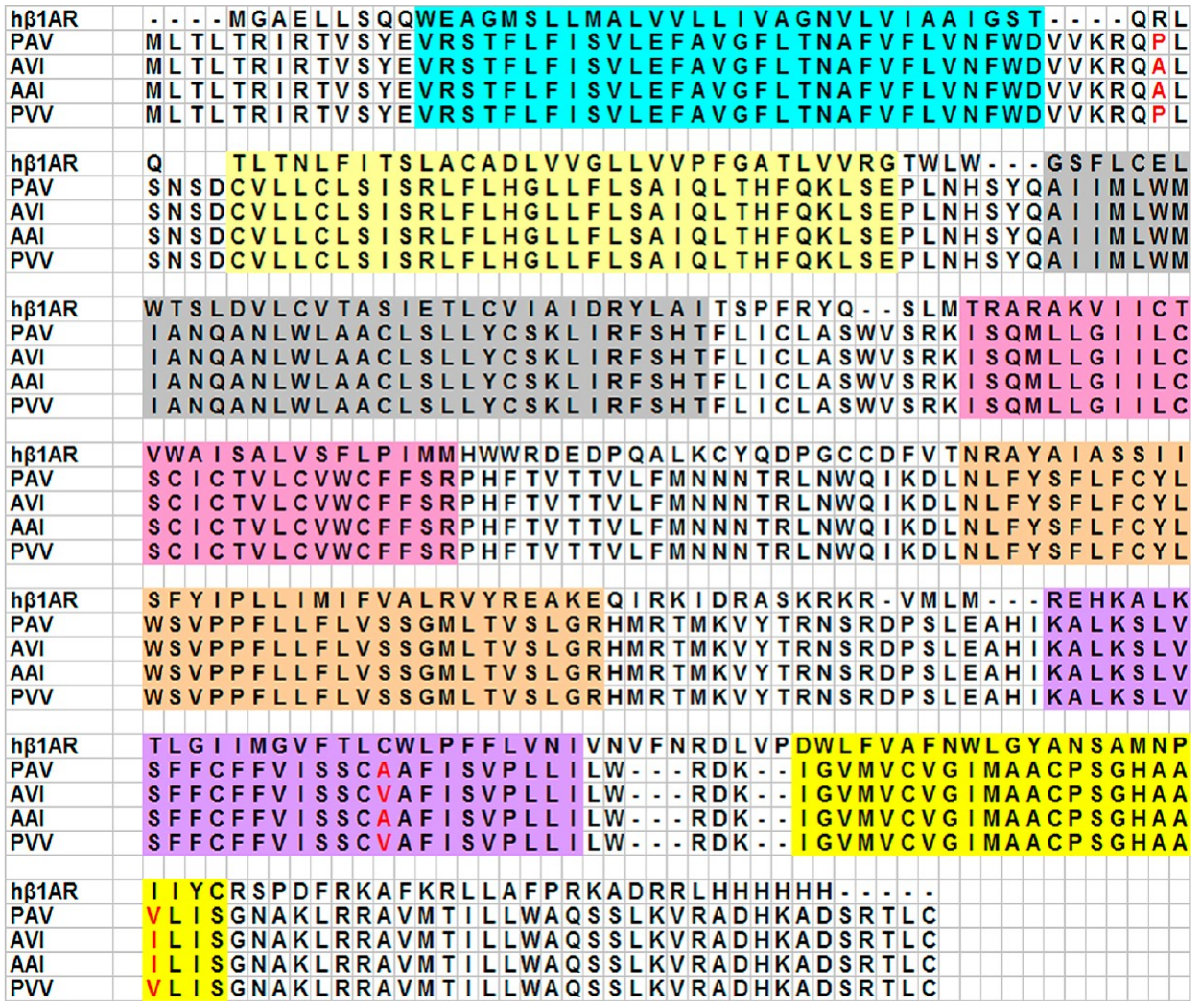

Figure 2. Sequence alignment against the $\beta 1$ adrenergic receptor $(\mathrm{h} \beta 1 \mathrm{AR})$ for TAS2R38 bitter taste receptor variants hTAS2R $38_{\mathrm{PAV}}, \mathrm{hTAS} 2 \mathrm{R} 38_{\mathrm{AAJ}}$ hTAS2R $38_{\mathrm{PVV}}$ (taster), and hTAS2R38 $8_{\mathrm{AVI}}$ (nontaster).
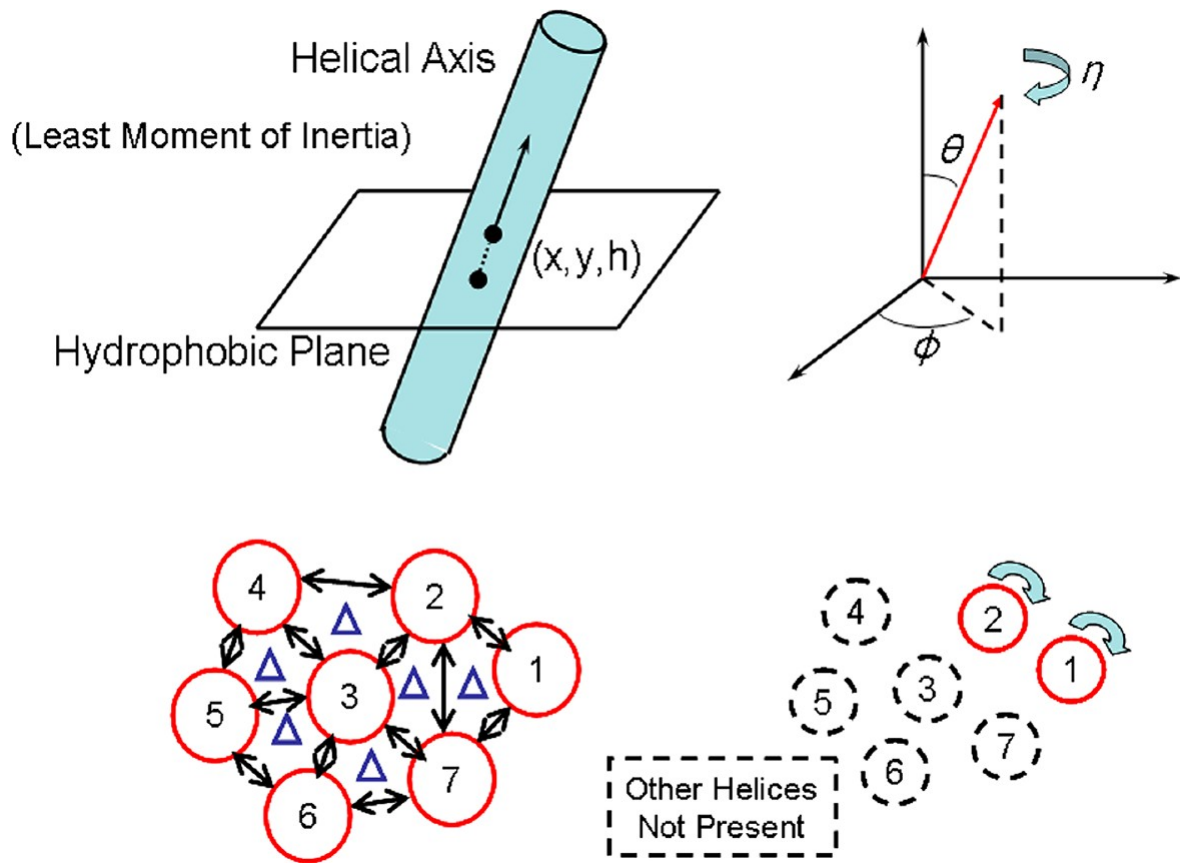

Figure 3. The coordinates used to describe the orientation of the seven helices in a GPCR bundle. Double arrows connecting nearest neighbor helix pairs that are sampled independently in the BiHelix procedure. The BiHelix procedure is highlighted using helices 1 and 2 to show that when the conformations for this helix pair are sampled, other helices are not present. In SuperBiHelix, the seven-helix bundles are partitioned into the three quadhelix bundles.

been detected in the lungs, suggesting that these receptors may play an important role in monitoring or controlling various physiological processes, with yet to be determined functions.
PTC is intensely bitter for some individuals but is largely tasteless for others, which has been traced to 2 common forms of the TAS2R38 gene which exhibit single-nucleotide polymorphisms (SNPs) at 3 sites: 
Table 1. ComBiHelix Results for Bitter Taste Receptors in the $\beta 1$ Adrenergic Receptor Template

\begin{tabular}{|c|c|c|c|c|c|c|c|c|}
\hline \multirow[b]{2}{*}{ receptor variants } & \multicolumn{7}{|c|}{ rotational angle } & \multirow[b]{2}{*}{ total energy $(\mathrm{kcal} / \mathrm{mol})$} \\
\hline & H1 & $\mathrm{H} 2$ & $\mathrm{H} 3$ & $\mathrm{H} 4$ & H5 & H6 & $\mathrm{H} 7$ & \\
\hline hTAS2R38 $8_{\text {PAV }}$ & 30 & 330 & 60 & 90 & 180 & 270 & 30 & 598.1 \\
\hline hTAS2R38 & 30 & 330 & 60 & 90 & 330 & 270 & 30 & 668.9 \\
\hline hTAS2R38 & 30 & 330 & 60 & 90 & 240 & 270 & 30 & 584.1 \\
\hline hTAS2R $38_{\mathrm{PVV}}$ & 30 & 330 & 60 & 90 & 240 & 270 & 30 & 597.4 \\
\hline
\end{tabular}

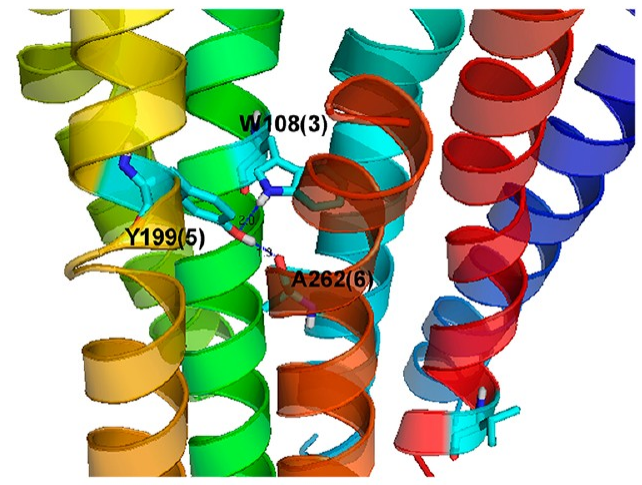

a

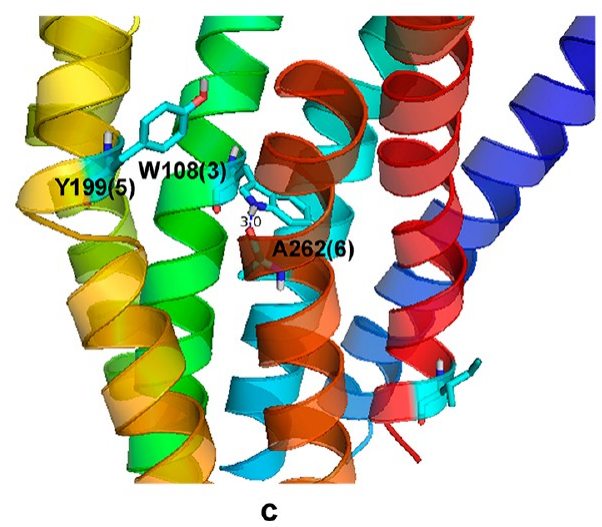

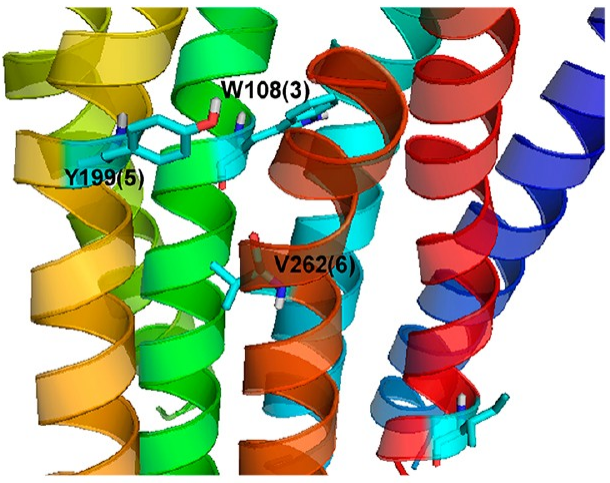

b

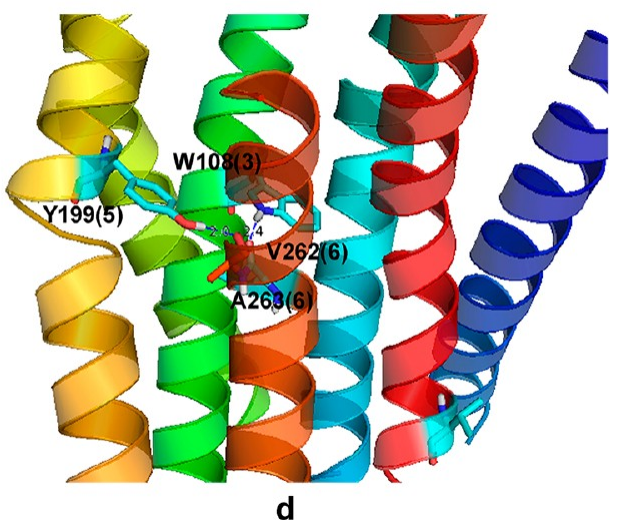

Figure 4. Predicted 3D structures of bitter taste receptors hTAS2R $38_{\text {PAV }}(a), h T A S 2 R 38_{\text {AVI }}$ (b), hTAS2R $38_{\text {AAI }}(c)$, and hTAS2R3 $8_{\text {PVV }}(d)$ from SuperComBiHelix (see Table 2). (Residues forming interhelical H-bonds are highlighted here).

- amino acid (AA) position 49, where either Pro or Ala is encoded,

- AA position 262, where either Ala or Val is encoded, and

- AA position 296, where either Val or Ile is encoded,

This gives rise to two frequent haplotypes, hTAS2R $38_{\text {PAV }}$ and hTAS2R $38_{\text {AVI }}$ plus less common haplotypes hTAS2R $38_{\mathrm{AAI}}$, hTAS2R $38_{\mathrm{PVI}}$, and hTAS2R38 ${ }_{\mathrm{AAV}}{ }^{13,14}$ where

- hTAS2R38 2 PAV shows a strong response at micromolar concentrations for PTC or PROP,

- hTAS2R38 $8_{\mathrm{AVI}}$ does not respond to PTC or PROP.

Mutational analysis ${ }^{14}$ determined that the amino acids at position 49 and 262 most affect the cellular response.

Several previous computational studies ${ }^{15-17}$ performed structural modeling of bitter taste receptors using homology structures based on bovine rhodopsin and considered binding of PTC or PROP to these bitter receptors. However, only limited insights were provided about the molecular mechanism of bitter taste sensing. In particular, these $3 \mathrm{D}$ structures ${ }^{15-17}$ found that residue 262 points toward the TM7 of bitter taste receptors and does not interact with the PTC agonist, making any correlation with experimental data ${ }^{14}$ difficult to interpret. To address the differences between bitter taster and nontaster TAS2R38 protein structures and the activation mechanism of TAS2R38 by agonists PTC and PROP (Figure 1), we used the BiHelix and SuperBiHelix Monte Carlo methods developed recently at $\mathrm{Caltech}^{18}$ to predict the 3D structures of the bitter taste receptors haplotypes hTAS2R38 $8_{\text {PAV }}$ (taster), hTAS2R38 $8_{\mathrm{AVI}}$ (nontaster), hTAS2R38 $8_{\mathrm{AAI}}$ (taster), and hTAS2R38 $8_{\text {PVV }}$ (taster). These methods are described in section 2, and the results for the apoprotein structures are described in section 3 .

Section 4 describes the DarwinDock Monte Carlo procedures we used to predict the binding sites and binding affinity for PTC and PROP to the predicted 3D structures of the various hTAS2R38 mutants, while section 5 analyzes the details of the binding (the cavity analysis) of these predicted ligand-protein complexes. Section 6 then discusses these results to extract an explanation for activation of bitter taste receptors that agrees with experimental data. ${ }^{14}$

\section{METHODS}

Methods for Predicting the 3D Structure for TAS2R38. Alignment. We carried out the PredicTM multiple sequence alignment among all taste receptors and also added turkey $\beta 1$ and 
$\widehat{\overline{\mathrm{g}}}$

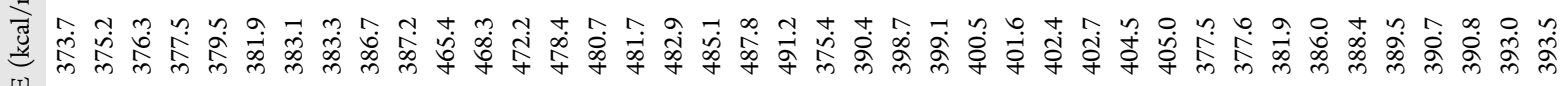
峞

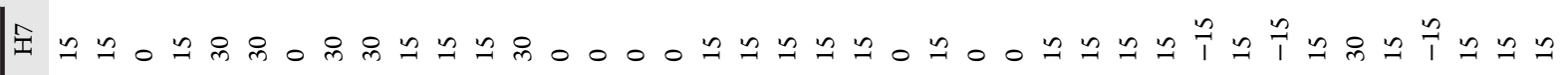

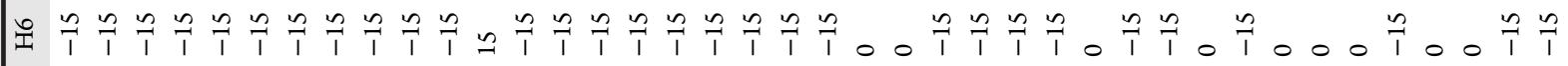
闰 0000000000 p

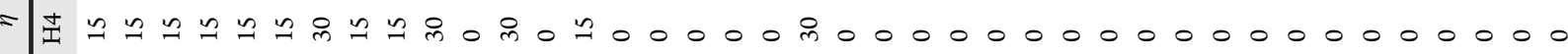

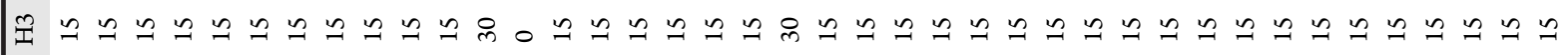
종

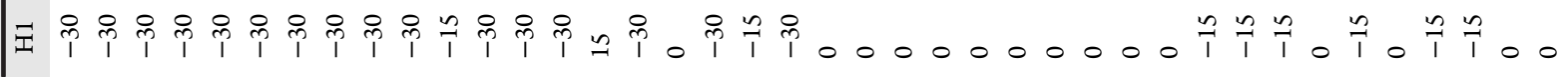

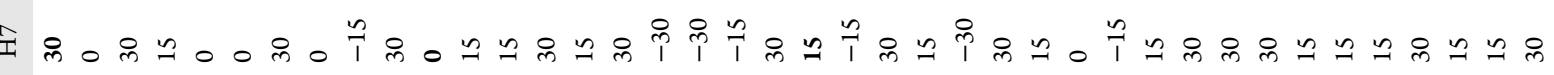

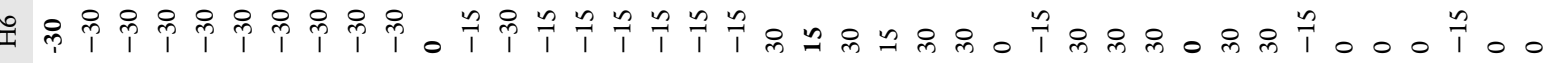

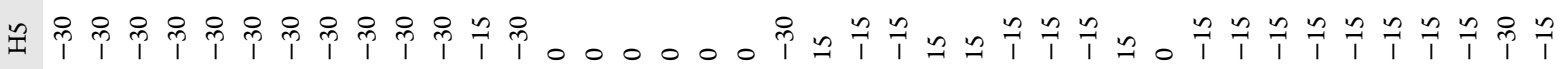
声㐱

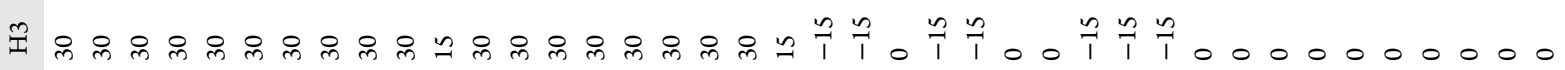

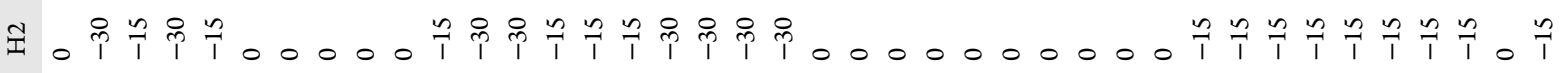

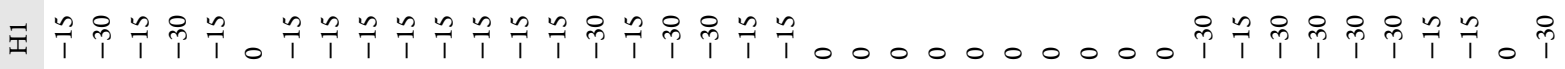

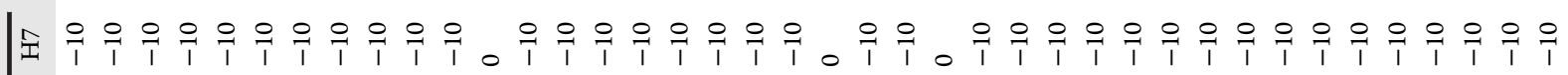

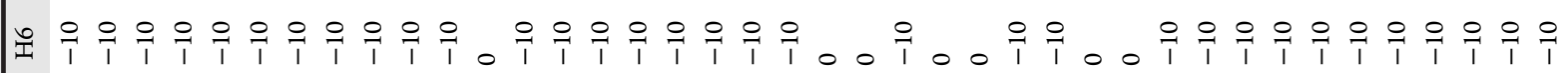

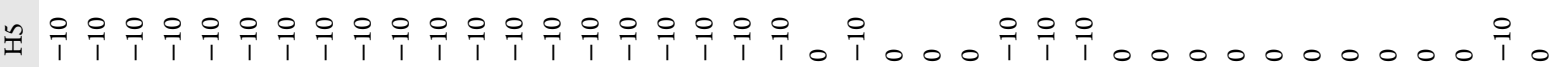

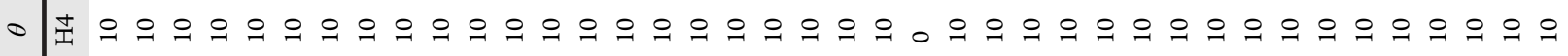

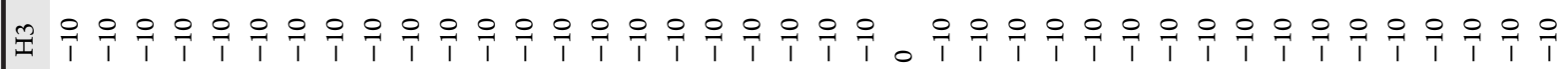

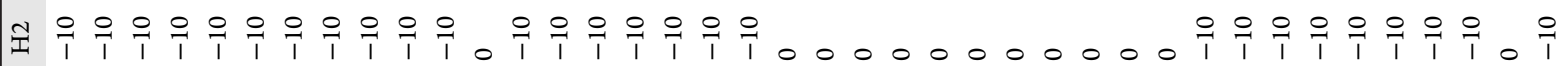

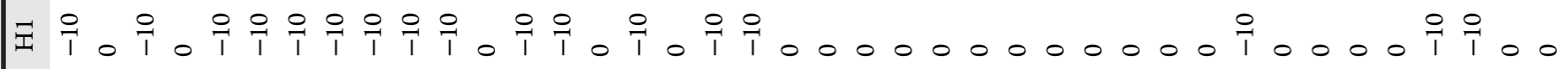
吾-nm+n 

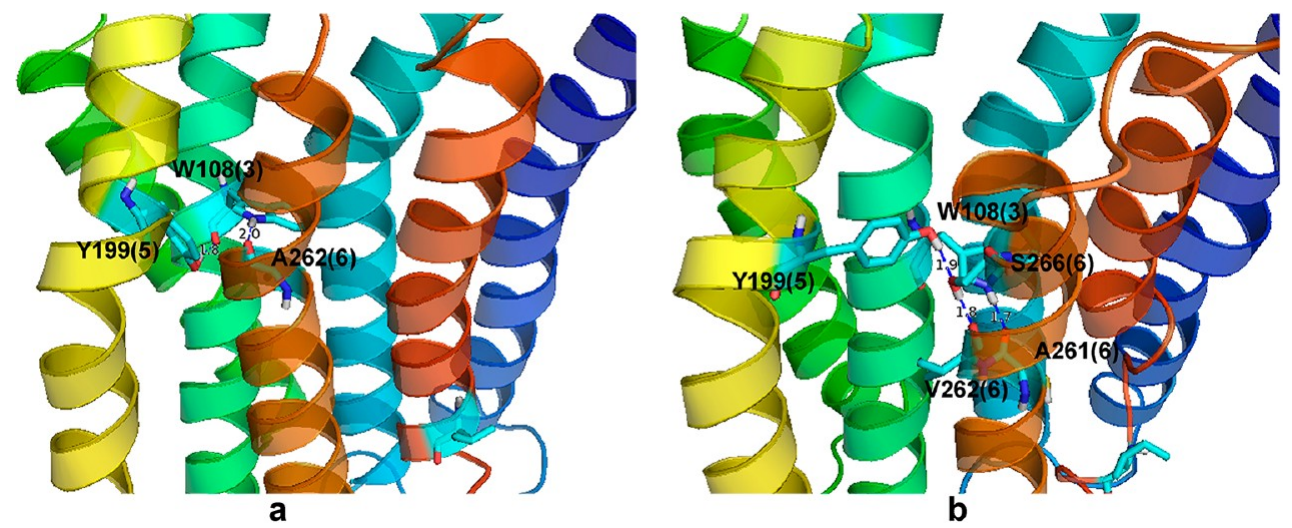

Figure 5. Predicted 3D structures of bitter taste receptors hTAS2R38 $8_{\text {PAV }}$ (a) and hTAS2R38 $8_{\text {AVI }}$ (b) after 10 ns MD with lipid and water. (Residues forming interhelical H-bonds are highlighted here, and they are stable during $10 \mathrm{~ns} \mathrm{MD}$ ).

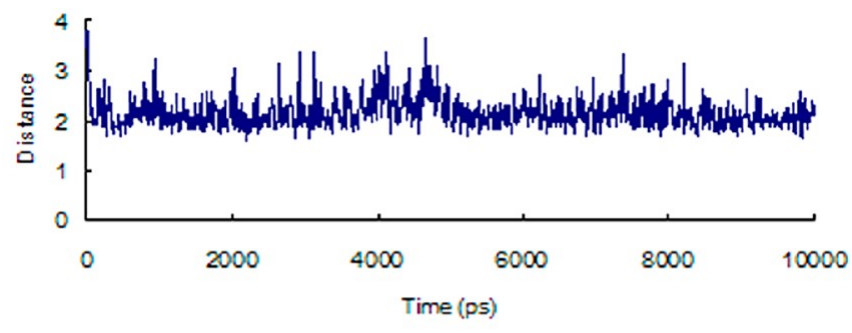

a

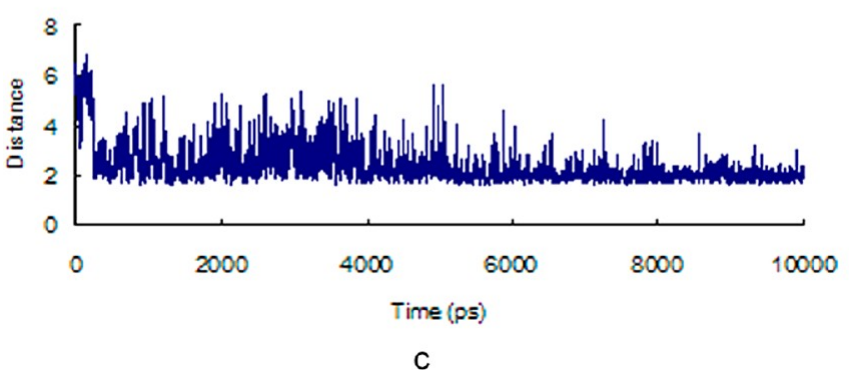

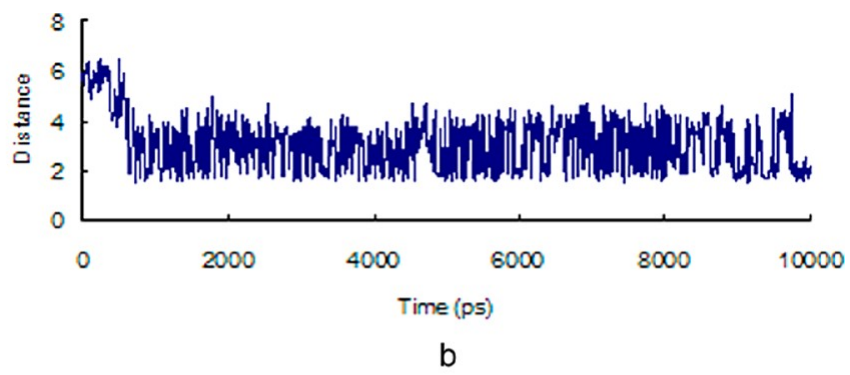

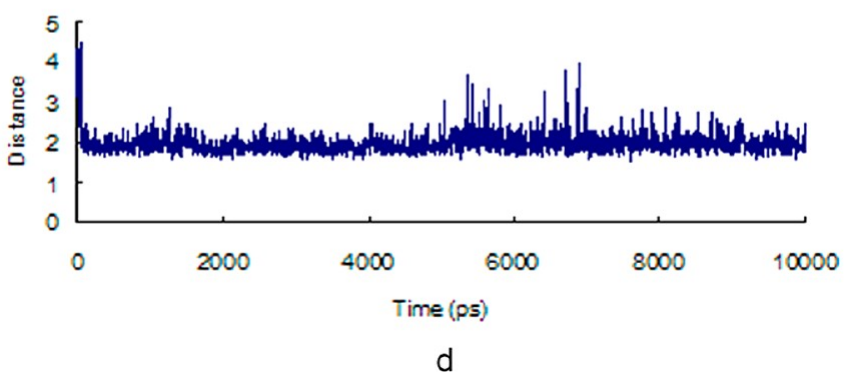

Figure 6. The stability of the predicted hydrogen bonds (HB) in bitter taste receptors over $10 \mathrm{~ns}$ MD with full lipid and water: (a) W108-A262 in hTAS2R38 $8_{\text {PAV }}$, (b) Y199-W108 in hTAS2R38 $8_{\text {PAV }}$, (c) Y199-A266 in hTAS2R38 AVV $_{\text {, }}$ and (d) W108-A261 in hTAS2R38 $8_{\text {AVI. }}$.

human $\beta 2$, adrenergic receptor ( $\mathrm{t} \beta 1 \mathrm{AR}$ and $\mathrm{h} \beta 2 \mathrm{AR}), \mathrm{A} 2 \mathrm{a}$ adenosine receptor $\left(\mathrm{hAA} \mathrm{A}_{2 \mathrm{~A}} \mathrm{R}\right.$ ), and bovine rhodopsin (BovR) as shown in Figure S1 of the Supporting Information (SI). The comparison between $\beta 1$ and TAS2R38 is shown in Figure 2 . PredicTM leads to a prediction of 7 hydrophobic regions expected to be in the membrane as indicated in Figure S2 of the SI.

Construction of Starting Structures from Multiple Templates. We mutated the residues of $\mathrm{h} \beta 1 \mathrm{AR}$ to the aligned sequences of TAS2R38 receptors and generated the initial 3D structure of TAS2R38 receptors. The same procedure was also carried out for $h \beta 2 A R, h A A_{2 A} R$, and BovR to generate starting structures based on different templates from crystal structures. Each experimental template has 42 degrees of freedom: $\mathrm{x}, \mathrm{y}, \mathrm{h}, \theta$, $\phi$, and $\eta$ values for each of the seven TM helices $(6 \times 7=42$ total). " $x$ " and " $y$ " determine the position of the helix on the $z=0$ plane. The hydrophobic center " $h$ " is the residue that crosses $z=0$, which is defined as the plane that runs through the center of the lipid bilayer. It is either calculated from the hydrophobic profile from PredicTM or by homology. The three orientation angles are the tilt angle $\theta$ of the helix, the sweep angle $\phi$ of the helix, and the rotation angle $\eta$ of the helix around the helical axis.

BiHelix. To determine the optimum rotation angle $\eta$ for each of the 7 helices, we allow each of the 7 helices to take on 12 orientations ( $30^{\circ}$ increments) about their axes. This leads to $12^{7}$ ( $\sim 35.83$ million) packings of the seven helices of the GPCR. The BiHelix procedure estimates the energies of these 35.83 million packings using a mean field constructed by considering the 12 sets of nearest neighbor bihelix interactions, TM1-TM2, TM1TM7, TM2-TM3, TM2-TM4, TM2-7, TM3-TM4, TM3-TM5, TM3-TM6, TM3-TM7, TM4-TM5, TM5-TM6, and TM6TM7. Here we use SCREAM to optimize the side chains for each of the 144 pairwise combinations of $\eta$ for each of the 12 pairs. $^{20}$ These 1728 pairwise energies are combined to estimate the energies for all 35.83 million packings from which we select the best 1000 for further analysis. The details of BiHelix sampling method have been described earlier. ${ }^{18}$

CombiHelix. We next build into a 7-helix bundle for the best 1000 results from BiHelix, reoptimize the side chains using SCREAM ${ }^{20}$ minimize, and reorder by energy. 
SuperBiHelix. For the optimum set of rotation angles $(\eta)$ from the previous step, we now sample a range of helix tilt angles $(\theta, \phi)$ simultaneous with $\eta$, to obtain the optimum 7-helix bundles. Again we consider the 12 pairs of strongly interacting helices but now accounting for the effect of tilts. Here we consider changes in $\theta$ by $\pm 15^{\circ}$ and changes in $\phi$ by $\pm 10^{\circ}$ and $\pm 20^{\circ}$, simulations with changes in $\eta$ by $\pm 15^{\circ}$ and $\pm 30^{\circ}$. Thus we sample $(3 \times 5 \times 5)^{7}=$ $13,348,388,671,875$ packings. These packings are analyzed by partitioning the seven-helix bundle into the three quadhelix bundles shown in Figure 3, to obtain 2000 structures with the lowest energy for each quadhelix. Then from each individual helical conformation list, the best 36 conformations for each helix are used to estimate the energy of $36^{7} \approx 8 \times 10^{10}$ full bundles. Finally we output the 1000 combinations estimated from this procedure to have the lowest energies.

SuperComBiHelix. These top 1000 helical bundles from SuperBiHelix are built into 7-helix bundles, and the side chains are reoptimized with SCREAM. ${ }^{20}$ Then the structure is minimized for 10 steps. This procedure results in an ensemble of 100 low energy bundles that may play a role in binding various ligands and in activation.

Prediction of the Extracellular (EC) and Intracellular (IC) Loop Structure. To provide initial structures for the three EC and IC loops for use in MD studies of the bitter taste receptors, we used the alignment of bitter taste receptor with $\mathrm{t} \beta 1 \mathrm{AR}$ and then homology threaded these loops to the crystal structure. Then, we carried out minimization and dynamics on the loops with fixed helix bundle atoms. We expect these loops to be quite flexible and strongly affected by the solvent, which was treated only implicitly in the previous steps.

Methods for Docking Ligands to the Predicted Protein Structures. We chose the top 10 structures of the apo-TAS2R38 based on total energy from SuperBiHelix step and docked both agonists PTC and PROP to each one. The geometry and charges of PTC and PROP were calculated using quantum mechanics (B3LYP with the 6-311G** basis set). We used two PTC conformers and four PROP conformers (Figure S5) for docking, as shown in the SI. We used the DarwinDock method to select ligand-binding conformations and to calculate their binding energies.

ScanBindSite. The whole protein was partitioned into 32 cubic regions (each with sides of $10 \AA$ ) and scanned to find the putative binding regions (with the six hydrophobic residues, I, L, V, F, Y, and W alanized). Here we used DOCK $4.0^{21}$ to generate 1000 conformations in each of the 32 putative regions and select 2 regions for further study based on a combination of burial score and binding energy. These 2 optimum regions were combined into a single region for docking.

Darwindock. We then generated $10,000-50,000$ poses using DOCK6.0, which were clustered into families in increments of 5,000 until $<2 \%$ new families are generated. The family heads were scored using the DREIDING $\mathrm{FF}^{22}$ and all members of top $10 \%$ family heads by energy are also scored. The top 100 poses from these (by energy) were selected, dealanized (replaced back the original hydrophobic residues), SCREAMed, ${ }^{20}$ and then minimized. Then we selected these for further minimization of the binding site complex (using the unified binding site including all residues within $4 \AA$ from any of the 10 binding poses). The protein and ligand were then neutralized by transferring protons appropriately in salt bridges and protonating or deprotonating exposed side chains (this leading to more reliable energy comparisons). ${ }^{23}$ Then the final docked structure with the best binding energy was selected.

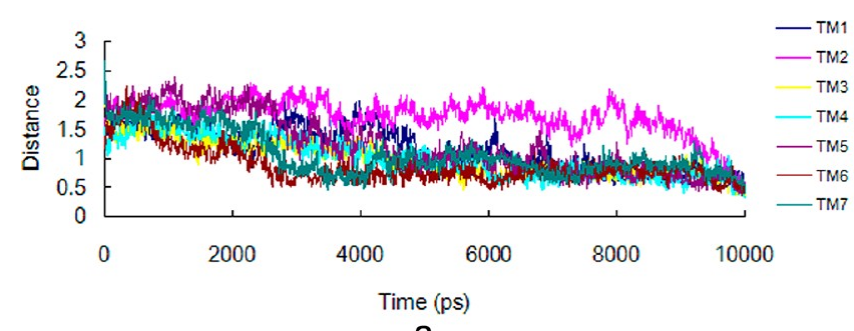

a

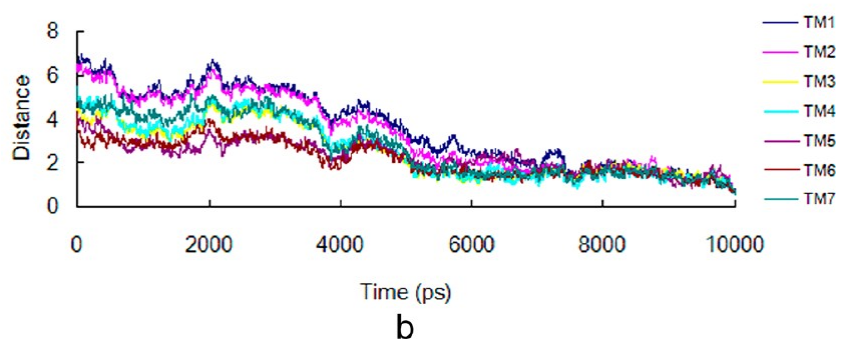

Figure 7. rmsd evolution of each helix of hTAS2R $38_{\text {PAV }}$ (a) and hTAS2R $38_{\mathrm{AVI}}$ (b) during $10 \mathrm{~ns} \mathrm{MD}$ (the reference is the last frame).

\section{RESULTS AND DISCUSSION}

Apo-Protein Structures for the Various Mutants. Selection of the Best Template. We shortened or lengthened the templates TMs and predicted the $3 \mathrm{D}$ structures of the TAS2R38 bitter receptors based on the four receptors templates $\mathrm{t} \beta 1 \mathrm{AR}, \mathrm{h} \beta 2 \mathrm{AR}$, BovR, and $\mathrm{hAA} \mathrm{A}_{\mathrm{A}} \mathrm{R}$, respectively. Figure $\mathrm{S} 1$ shows the TMs sequence alignments between PAV and four templates $\left(\mathrm{t} \beta 1 \mathrm{AR}, \mathrm{h} \beta 2 \mathrm{AR}, \mathrm{BovR}\right.$, and $\left.\mathrm{hAA} \mathrm{A}_{2 \mathrm{~A}} \mathrm{R}\right)$ which are used to build the $3 \mathrm{D}$ structures by the homology modeling. After minimization, the initial structures combining the seven TM segments from the four templates (without loops and the eighth helices) were built. After BiHelix and ComBiHelix, the rotational angles of seven TMs were generated, and top 2000 conformations of bitter taste receptors were obtained according to the total energy. Table $\mathrm{S} 1$ shows the best conformations of PAV receptor with the best total energy from $\mathrm{BiHelix}$ and $\mathrm{ComBiHelix}$ results. The helix -6 rotational angle of the structure based on $\mathrm{t} \beta 1 \mathrm{AR}$ template is larger than those based on other templates. The structure based on $\mathrm{t} \beta 1 \mathrm{AR}$ template has the lowest total energy compared with those based on other templates. Figure S3 shows that residue $\mathrm{A} 262$ points inside the bundles based on $\mathrm{t} \beta 1 \mathrm{AR}$, while it points outside in the bundles based on $\mathrm{h} \beta 2 \mathrm{AR}$ and toward TM7 of the structure based on rhodopsin and hAA ${ }_{2 \mathrm{~A}} \mathrm{R}$ templates. It has been reported that the residue 262 in hTAS2R38 bitter taste receptor is very important for the bitter taste. ${ }^{14}$ This suggests that the residue 262 is located inside the TMs bundle so that it can interact with the agonist and/or other helices during activation. This is consistent with our finding that $\mathrm{t} \beta 1 \mathrm{AR}$ template led to the lowest energy conformations and provides a preliminary validation of the predicted structures.

Comparison of the $3 D$ Structure of Various Haplotypes of TAS2R38 Bitter Receptors. We constructed the structural models of the TAS2R38 bitter receptors (four haplotypes hTAS2R38 $8_{\text {PAV }}$, hTAS2R $38_{\text {AVV }}$, hTAS2R $38_{\text {AAl }}$ and hTAS2R $38_{\text {PVV }}$ ) using $\mathrm{t} \beta 1$ AR as a template. The ensemble of conformations with different helical rotation angles $\eta$, tilt angles $\theta$, and sweep angles $\phi$ were generated based on BiHelix and SuperBihelix methodology described earlier. ${ }^{18}$ We discuss here the details only for the best (the lowest total energy) predicted protein structures.

Figure 2 shows the TMs sequence alignments between hTAS2R38 $8_{\text {PAV }}$, hTAS2R38 $8_{\text {AVI }}$, hTAS2R38 $8_{\text {AAI }}$, hTAS2R38 $8_{\text {PVV }}$, 

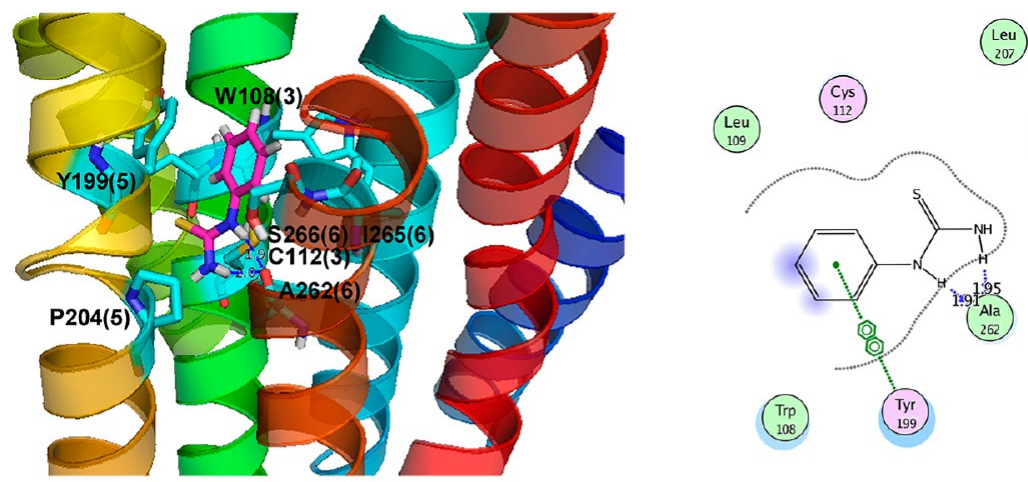

Pro
204

Val

a

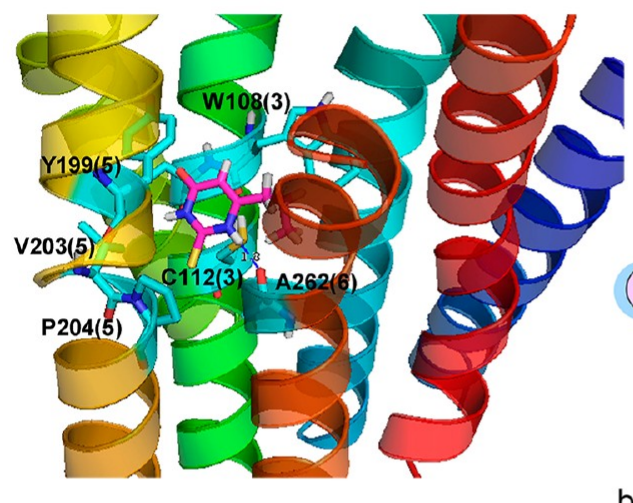

val 203

Pro<smiles>C=C1NC(=O)C=C(CCC)N1c1ccccc1</smiles>

(Cys

(112)

b
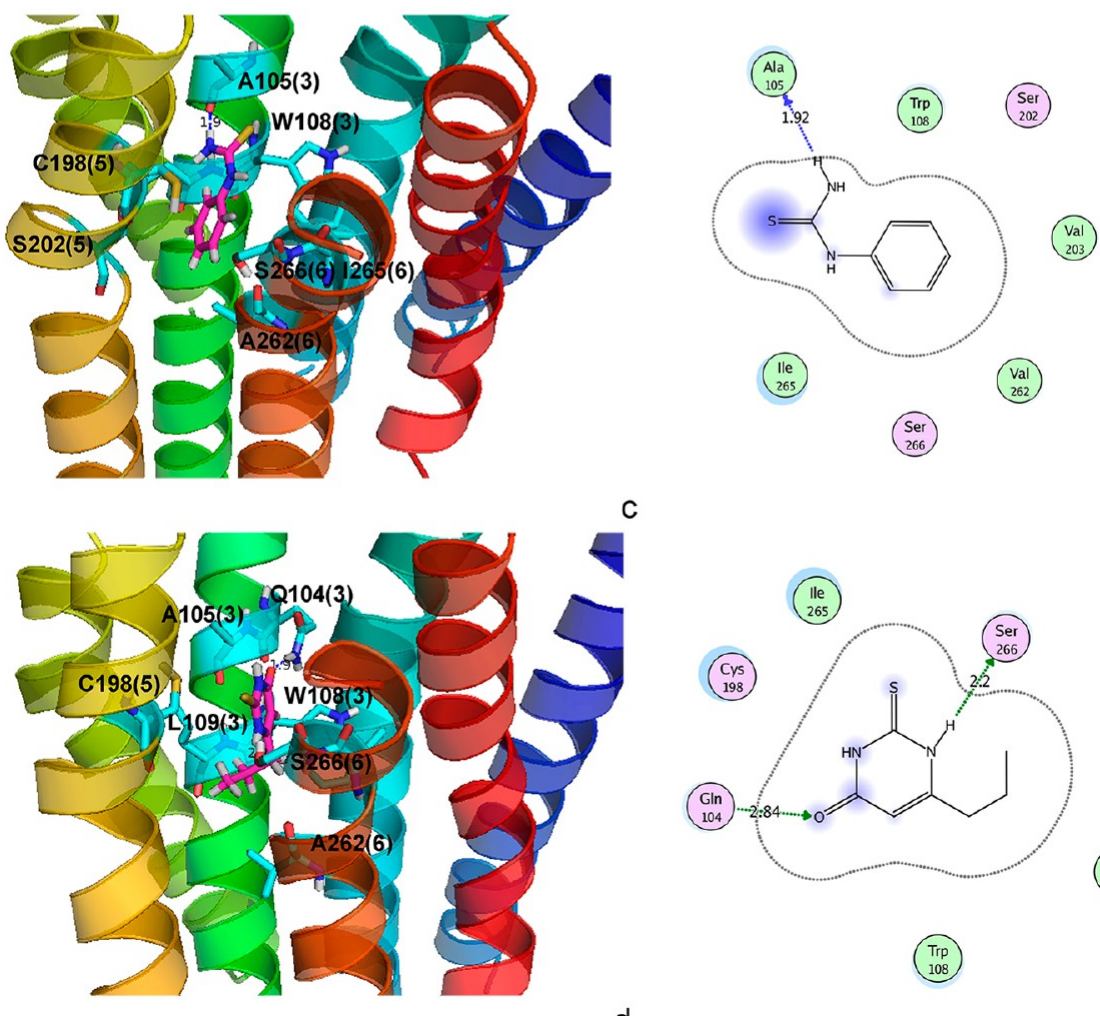

val

val

Figure 8. Predicted binding sites of agonists in bitter taste receptors: (a) PTC in hTAS2R38 $8_{\text {PAV }}$, (b) PROP in hTAS2R38 $8_{\text {PAV }}$, (c) PTC in hTAS2R38 $8_{\text {AVI }}$ and (d) PROP in hTAS2R38 $8_{\text {AVI }}$. The green one is nonpolar hydrophobic amino acid, while the purple one is polar amino acid in the $2 \mathrm{D}$ digram.

and $\mathrm{h} \beta 1 \mathrm{AR}$ which are used to build the $3 \mathrm{D}$ structures. As shown in Table 1, BiHelix results suggest that the helices of four variants have identical rotational angles except for helix 6.
The TAS2R38 bitter receptors lack some of the wellconserved motifs present in class A GPCRs. Thus we can expect that the TAS2R38 bitter receptors might have a different set of 
stabilizing interhelical hydrogen bonds when compared to $\mathrm{h} \beta 1 \mathrm{AR}$. The predicted 3D structures of four variants of the TAS2R38 bitter receptors are shown in Figure 4, and the residues forming interhelical H-bonds are highlighted.

We find the interhelical hydrogen bonds between Y199 (5) and W108 (3) and between Y199 (5) and A262 (6) in hTAS2R38 $8_{\text {PAV }}$ protein. An interhelical bond between W108 (3) and A(V)262 (6) exists in both hTAS2R38 $8_{\mathrm{AAI}}$ and hTAS2R38 $8_{\mathrm{PVV}}$ protein. These interhelical bonds do not exist in the hTAS2R $38_{\text {AVI }}$ (nontaster) protein.

To address the structural stability of these four variants, we perform $10 \mathrm{~ns}$ of molecular dynamics simulation on the hTAS2R38 $8_{\text {PAV }}$ and hTAS2R38 $8_{\text {AVI }}$ protein structures in a full-atom lipid bilayer environment (see the the SI for details of these MD studies). Figure 5 shows the $3 \mathrm{D}$ structures of the hTAS2R38 $8_{\text {PAV }}$ and hTAS2R $38_{\mathrm{AVI}}$ proteins after $10 \mathrm{~ns} \mathrm{MD}$ with lipid and water. The hydrogen bonds in the hTAS2R $38_{\text {PAV }}$ protein were broken, and two new hydrogen bonds between Y199 (5) and W108 (3) and between W108 (3) and A262 (6) are formed that were also observed in hTAS2R $38_{\mathrm{AAI}}$ and hTAS2R $38_{\mathrm{PVV}}$ receptors. We also find 2 new interhelical hydrogen bonds between W108 (3) and

Table 3. Energy Analysis for PTC and PROP Docked to the Predicted hTAS2R38 ${ }_{\text {PAV }}$ and hTAS2R38 $8_{\text {AVI }}$ Proteins

\begin{tabular}{clcccc} 
receptor & ligand & $\begin{array}{c}\text { unified } \\
\text { cavity } \\
(\mathrm{kcal} / \mathrm{mol})\end{array}$ & $\begin{array}{c}\text { local cavity } \\
(\mathrm{kcal} / \mathrm{mol})\end{array}$ & $\begin{array}{c}\text { binding } \\
\text { energy } \\
(\mathrm{kcal} / \mathrm{mol})\end{array}$ & $\begin{array}{c}\text { total energy } \\
(\mathrm{kcal} / \mathrm{mol})\end{array}$ \\
PAV & PTC & -27.24 & -25.22 & -33.60 & -54.06 \\
& PROP & -25.66 & -23.15 & -31.31 & -50.52 \\
\multirow{2}{*}{ AVI } & PTC & -24.23 & -22.59 & -31.94 & -70.11 \\
& PROP & -26.13 & -24.78 & -38.54 & -80.00
\end{tabular}

A261 (6) and between Y199 (5) and A266 (6) in hTAS2R38 protein. Figure 6 shows that these new H-bonds are all maintained during the $10 \mathrm{~ns} \mathrm{MD}$.

Figure 7 shows the $\mathrm{C} \alpha$ root-mean-square deviation (CRMSD) time evolution of the helical segments during the $10 \mathrm{~ns}$ MD. Here CRMSD is with respect to the last frame of the $10 \mathrm{~ns}$ trajectory. The total range of the CRMSD from the initial predicted structure to the final one in the trajectory ranges for $0.5-2.5 \AA$ in hTAS2R $38_{\mathrm{PAV}}$ and for 1.0-7.0 ̊̊ in hTAS2R38 $8_{\mathrm{AVI}}$. Focusing on the last $2 \mathrm{~ns}$, these fluctuations range from 0.5 to $1.5 \AA$, except TM2 in hTAS2R38 $8_{\text {PAV }}$ which reaches 2.0 A.

Binding Sites for Agonist PTC and PROP. We used the DarwinDock technique to predict the binding site of agonists to the four predicted (lowest energy) structures of TAS2R38 bitter receptors. The predicted binding sites of agonist PTC and PROP in hTAS2R38 $8_{\text {PAV }}$ and hTAS2R38 $8_{\text {AVI }}$ receptors are shown in Figure 8. PTC and PROP binding site is located between the TM 3, 5, and 6 helices. The most important residues (cavity analysis) are (including the interaction energy with ligands in parentheses) as follows:

hTAS2R38 PAV with PTC: ALA 262(-5.336), TYR199(-5.209), CYS 112(-2.68), TRP 108(-2.579), LEU 109(-2.564), ILE 265(-1.235), PRO 204(-1.106), SER 266(-0.976).

hTAS2R38 ${ }_{\text {PAV }}$ with PROP: ALA262 (-4.085),TYR199 (-2.975), TRP108 (-2.127),CYS112 (-1.746), VAL203 $(-1.699)$, LEU109 (-1.655), ALA263 (-1.155), PRO204 (-1.056).

hTAS2R38 $8_{\text {AVI }}$ with PTC: ALA105 (-4.637), TRP108 (-3.035), CYS198 (-2.410), ILE265 (-1.982), LEU109 (-1.937), VAL262 (-1.525), SER202 (-1.289), SER266 $(-0.999)$.
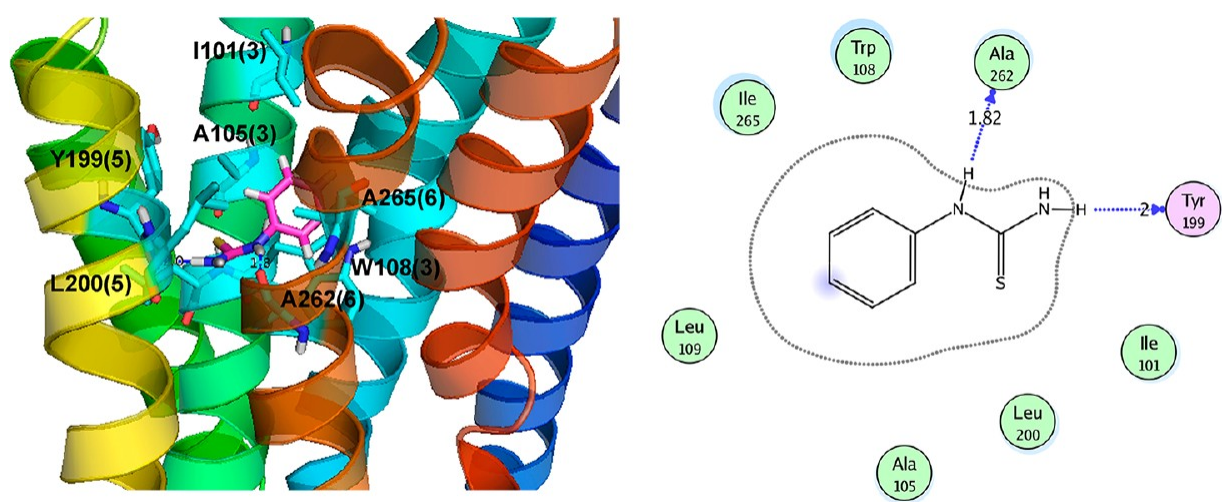

a
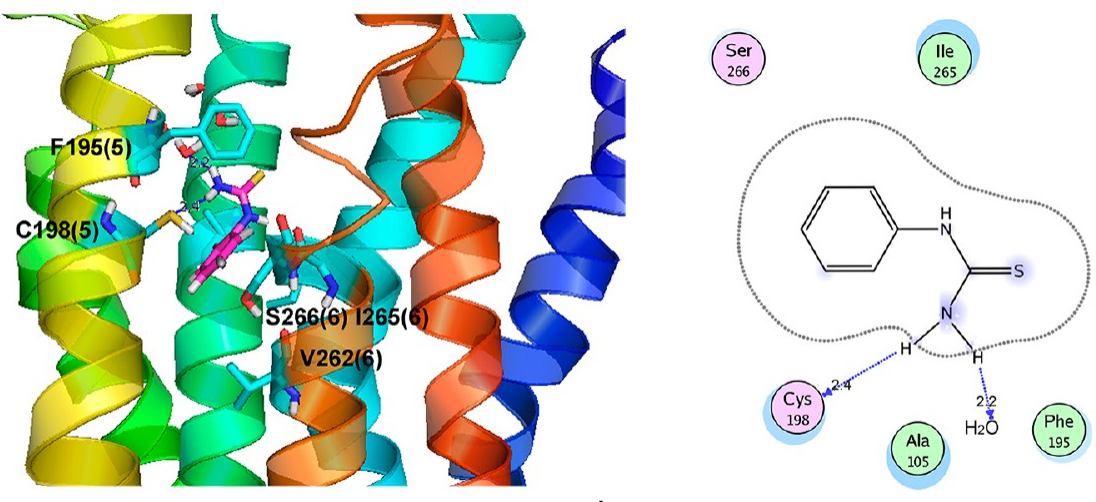

b

Figure 9. The final binding sites of PTC in hTAS2R38 $8_{\text {PAV }}(\mathrm{a})$ and hTAS2R $38_{\text {AVI }}$ (b) after $10 \mathrm{~ns}$ MD with lipid and water. The essential elements of the binding mode are retained, but additional favorable interactions are found. 


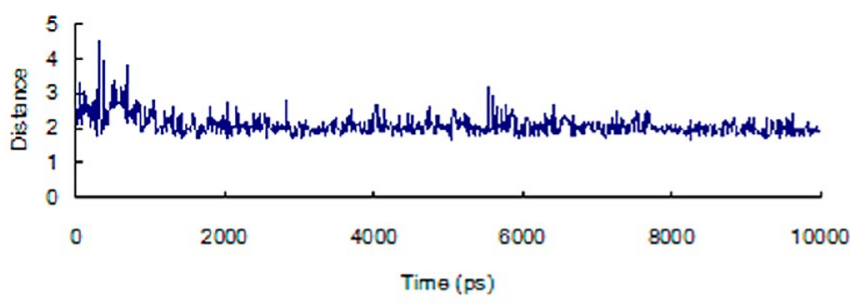

a

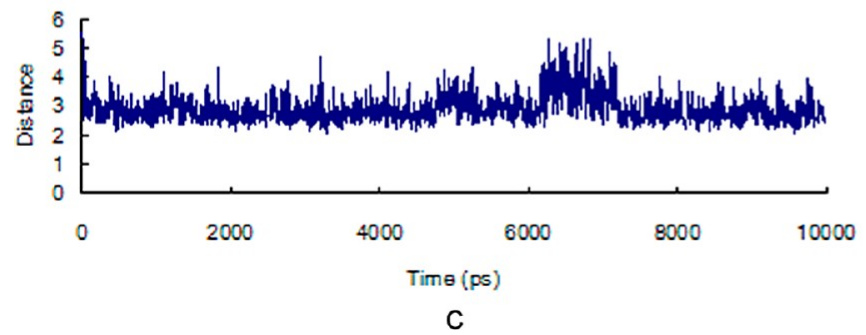

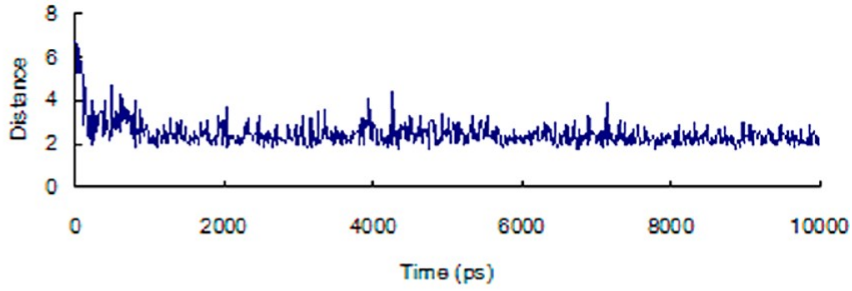

b

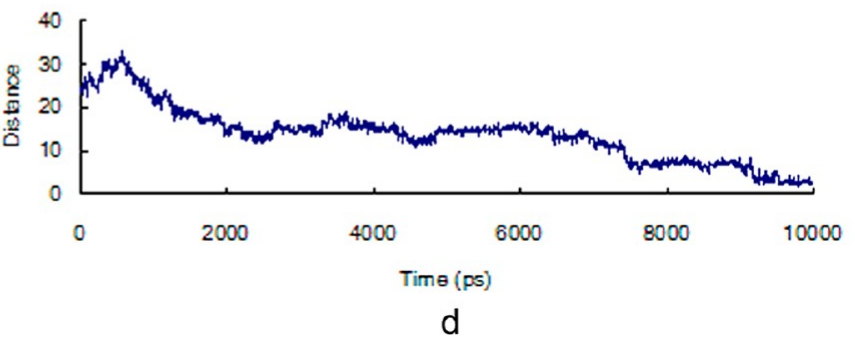

Figure 10. The H-bond distances bonding PTC to hTAS2R $38_{\mathrm{PAV}}$ and hTAS2R $38_{\mathrm{AVI}}$ during the $10 \mathrm{~ns}$ molecular dynamics with lipid and water: (a) PTC-A262 in hTAS2R38 $8_{\text {PAV; }}$ (b) PTC-Y199 in hTAS2R38 ${ }_{\text {PAV; }}$ (c) PTC-C198 in hTAS2R38 $8_{\text {AVI; }}$ and (d) PTC-water in hTAS2R38 ${ }_{\text {AVI. Note in }}$ particular the formation of the last three hydrogen bonds $(b, c$, and $d$ ) not present in the original predicted binding site.

hTAS2R38 $8_{\text {AVI }}$ with PROP: SER266 (-4.047), GLN104 (-3.994), CYS198 (-3.075), TRP108 (-3.072), ILE265 (-2.243), VAL262 (-2.032), LEU109 (-1.965), ALA $105(-1.149)$.

As shown in Figure 8, there are two strong hydrogen bonds between PTC and ALA 262 in hTAS2R38 $8_{\mathrm{PAV}}$ and the $\pi-\pi$ interaction between PTC and TYR 199 in hTAS2R38 $8_{\text {PAV }}$. There is a hydrogen bond between PROP and ALA 262 in hTAS2R38 $8_{\text {PAV. }}$. In hTAS2R38 $8_{\text {AVI }}$ PTC interacts with ALA 105 by H-bond, while PROP interacts with SER 266 and GLN 104 by H-bonds. Furthermore, we docked PTC and PROP to other tasters hTAS2R38 and hTAS2R38 $\mathrm{PvV}$. The predicted binding sites illustrate that PTC and PROP have hydrogen bonds with the residue 262 (A or V) in both hTAS2R $38_{\mathrm{AAI}}$ and hTAS2R $38_{\mathrm{PVV}}$ as shown in Figure S6. These results suggest that the residue 262 is very important for bitter taste perception which agrees with experimental data. ${ }^{14}$

Table 3 shows the calculated binding energies for PTC and PROP docked to the predicted hTAS2R $38_{\text {PAV }}$ and hTAS2R $38_{\text {AVI }}$ proteins. PTC shows stronger binding for hTAS2R38 $8_{\text {PAV }}$ than PROP, which indicates that PTC is more sensitive to hTAS2R38 than PROP as reported by Bufe et al. ${ }^{14}$

After inserting the predicted hTAS2R $38_{\text {PAV }} /$ PTC and hTAS2R38 $8_{\mathrm{AVI}} /$ PTC complexes into the infinite lipid membrane and solvating fully with water (using the procedure described in the SI), we performed $10 \mathrm{~ns}$ of MD. Figure 9a and Figure 8a compare the structure of the hTAS2R38 $8_{\text {PAV }} /$ PTC complex after $10 \mathrm{~ns}$ with the initial predicted structure. We find that the hydrogen bonds of the original predicted structures remain stable during $10 \mathrm{~ns}$ dynamics. However, one additional hydrogen bond is formed between the $-\mathrm{NH}_{2}$ group of PTC and Y199 (5) in

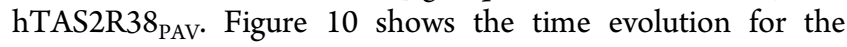
hydrogen bonds distances between PTC and hTAS2R38 $8_{\mathrm{PAV}}$.

(1) $-\mathrm{NH}-\mathrm{A} 262$ (6): the hydrogen bond distance remains mostly between 1.7 and $3.0 \AA$ except the occasional extensions to $4.9 \AA$ A during initial 10 ps.

(2) $-\mathrm{NH}_{2}-\mathrm{Y} 199$ (5): the initial distance is $6.6 \AA$, but it quickly contracts to $3.0 \AA$ and then mostly fluctuates between $2.0 \AA$ and $4.0 \AA$ and finally comes back to $\sim 2.5 \AA$.
Thus, we conclude that PTC forms strong interactions with both TM5 and TM6 in the hTAS2R38 $8_{\text {PAV }}$ structure. The binding of ligand between TM5 and TM6 breaks the strong coupling between TM3 and TM6 and between TM3 and TM5.

As shown in Figure 9b, the ligand in the structure of the hTAS2R38 $8_{\mathrm{AVI}} /$ PTC complex after $10 \mathrm{~ns}$ molecular dynamics moves up, compared with that of the initial predicted structure. So the initial $\mathrm{H}$-bond between ligand and $\mathrm{A} 105$ was broken, and a water molecule moves into the binding site and form $\mathrm{H}$-bonds with the ligand. The insertion of ligand in hTAS2R $38_{\text {AVI }}$ protein breaks interhelical hydrogen bonds between W108 (3) and A261 (6) while not that between Y199 (5) and A266 (6).

The Activation of Bitter-Taste Receptor. The protein structure prediction and MD simulation results indicate that the H-bonds between W108 (3) and A (or V) 262 (6) could stabilize the tasters hTAS2R38 $8_{\mathrm{PAV}}$, hTAS2R $38_{\mathrm{AAV}}$ and hTAS2R $38_{\mathrm{PVV}}$ and that the H-bonds between W108 (3) and A261 (6) stabilize the nontaster hTAS2R $38_{\mathrm{AVI}}$. We furthermore find that both PTC and PROP can interact with A (or V) 262 in tasters (hTAS2R38 $8_{\text {PAV }}$, hTAS2R $38_{\mathrm{AAV}}$, and hTAS2R $38_{\mathrm{PVV}}$ ) by hydrogen bond to their backbone, while there is no $\mathrm{H}$-bond between ligands and residue 262 in the nontaster (hTAS2R $38_{\text {AVI }}$ ). We outline here the difference between the agonist-bound taster and the agonist-bound nontaster. One of the major differences involves the local environment around residue position 262 in the hTAS2R38 receptor. The residue 262 is involved in not only the TM3-TM6 interaction but also the binding of agonist to receptors.

Fluorescence experiments on rhodopsin during activation show that TM3-TM6 interaction is involved in GPCR activation. ${ }^{24-26}$ The mutation experiments in TAS2R38 indicate that position 262 is important to taste bitter for the TAS2R38 bitter receptors. ${ }^{14}$ So our results indicate that the H-bonds between $\mathrm{W} 108(3)$ and $\mathrm{A}($ or V)262(6) and between agonists and $\mathrm{A}$ (or V)262(6) in tasters play a crucial role in GPCR activation and bitter-tasting, which is consistent with the experimental data. ${ }^{14}$

As shown in Table 1, in addition, the mutation of A262 V and V296I results in a larger rotation angle of TM5 in hTAS2R38 and make the Y199 closer to TM6 so that the formation of the hydrogen bond between Y199 and A266 could lead to the form of H-bond between W108 and A261 not V262. Although the 
mutation of A262 V exists in the hTAS2R38 $8_{\mathrm{PVV}}$, the smaller rotation angle of TM5 cannot cause the formation of TM5-TM6 hydrogen bond interaction. Thus the hydrogen bond interaction between TM3 and TM6 may pass the signal to intracellular to activate receptor.

Importantly, both PTC and PROP have $\mathrm{H}$-bonds with residue 262 of the tasters (hTAS2R38 $8_{\text {PAV }}$, hTAS2R38 $8_{\mathrm{AAI}}$ and hTAS2R38 ${ }_{\mathrm{PVV}}$ ) and not with that of nontaster (hTAS2R38 $\mathrm{AVI}$ ). MD simulation results suggest that PTC forms the stable H-bonds with Y199 in TM5 and A262 in TM6 of the hTAS2R38 $8_{\text {PAV }}$ structure, while PTC in hTAS2R38 $8_{\text {AVI }}$ prefers to move away from V262. So the residue 262, involved in the interaction between PTC and bitter taster structure, is very important to taste bitter compounds in agreement with experimental observations. ${ }^{14}$

\section{CONCLUSIONS}

The 3D structures of four haplotypes of TAS2R38 G-protein coupled receptors (GPCRs) were predicted to gain molecular level understanding of bitter taste receptors. We find the formation of H-bonds between W108 (3) and A262 (6) in hTAS2R38 $8_{\text {PAV }}$ (taster) and between W108 (3) and A261 (6) in hTAS2R $38_{\text {AVI }}$ (nontaster) could stabilize the protein structure. To further validate these structures, we used the DarwinDock method to predict the binding sites and $3 \mathrm{D}$ structures for PTC and PROP bound to hTAS2R38 $8_{\text {PAV }}$, hTAS2R $38_{\text {AVI }}$, hTAS2R38 $8_{\text {AAI }}$ and hTAS2R38 $8_{\text {PVV }}$, respectively. The predicted binding sites position PTC and PROP in the region between TM3, TM5, and TM6. The PTC and PROP could form the H-bonds with residue 262 in the tasters (hTAS2R38 $38_{\mathrm{PAV}}$, hTAS2R38 $38_{\mathrm{AAI}}$, and hTAS2R $38_{\mathrm{PVV}}$ ) and not with that in the nontaster (hTAS2R38 $8_{\text {AVI }}$ ). The H-bonds between PTC and A262 (6) and between PTC and Y199 in hTAS2R38 $8_{\text {PAV }}$ are stable. However, PTC in hTAS2R $38_{\text {AVI }}$ moves up away from V262 (6) during $10 \mathrm{~ns} \mathrm{MD.}$ So our results indicate that the hydrogen bond interaction between TM3 and TM6 may pass the signal to intracellular to activate receptor and that the $\mathrm{H}$-bond between agonists and residue 262 in tasters is involved in the bitter taste sensation, which agrees with the experimental result. ${ }^{14}$

\section{ASSOCIATED CONTENT}

\section{S Supporting Information}

Methods of molecular dynamics simulation, Table I, and Figures S1-S6. This material is available free of charge via the Internet at http://pubs.acs.org.

\section{AUTHOR INFORMATION}

\section{Corresponding Author}

*Phone: (626)395-2731. Fax: (626) 585-0918. E-mail: wag@ wag.caltech.edu.

\section{Present Addresses}

${ }^{\ddagger}$ Current address: Key Laboratory of Biorheological Science and Technology, Ministry of Education, Bioengineering College, Chongqing University, Chongqing, 400030, China.

\section{Notes}

The authors declare no competing financial interest.

\section{ACKNOWLEDGMENTS}

We thank Dr. Soo-Kyung Kim for helpful discussions and Jenelle Bray for use of the BiHelix and SuperBiHelix procedures prior to publication. J.T. was financially supported by National Natural Science Foundation of China (No. 20901086) and by NIH
(R01NS071112). Others were supported partially by NIH (R01NS071112) and by Sanofi-Aventis (SABER).

\section{REFERENCES}

(1) Lindeman, B. Taste reception. Physiol. Rev. 1996, 76, 718-766.

(2) Glendinning, J. I. Is the bitter rejection response always adaptive? Physiol. Behav. 1994, 56, 1217-1227.

(3) Behrens, M.; Foerster, S.; Staehler, F.; Raguse, J. D.; Meyerhof, W. Gustatory expression pattern of the human TAS2R bitter receptor gene family reveals a heterogeneous population of bitter responsive taste receptor cells. J. Neurosci. 2007, 27, 12630-12640.

(4) Bufe, B.; Hofmann, T.; Krautwurst, D.; Raguse, J. D.; Meyerhof, W. The human TAS2R16 receptor mediates bitter taste in response to betaglucopyranosides. Nat. Genet. 2002, 32, 397-401.

(5) Conte, C.; Ebeling, M.; Marcuz, A.; Nef, P.; Andres-Barquin, P. J. Identification and characterization of human taste receptor genes belonging to the TAS2R family, Cytogenet. Genome. Res. 2002, 98, 4553.

(6) Adler, E.; Hoon, M. A.; Mueller, K. L.; Chandrashekar, J.; Ryba, N. J.; Zuker, C. S. A novel family of mammalian taste receptors. Cell 2000, 100, 693-702.

(7) Chandrashekar, J.; Mueller, K. L.; Hoon, M. A.; Adler, E.; Feng, L.; Guo, W.; Zuker, C. S.; Ryba, N. J. T2Rs function as bitter taste receptors. Cell 2000, 100, 703-711.

(8) Lindemann, B. Receptors and transduction in taste. Nature 2001, 413, 219-225.

(9) Chandrashekar, J.; Hoon, M. A.; Ryba, N. J.; Zuker, C. S. The receptors and cells for mammalian taste. Nature 2006, 444, 288-294.

(10) Janssen, S.; Laermans, J.; Verhulst, P. J.; Thijs, T.; Tack, J.; Depoortere, I. Bitter taste receptors and $\alpha$-gustducin regulate the secretion of ghrelin with functional effects on food intake and gastric emptying. Proc. Natl. Acad. Sci. U.S.A. 2011, 108, 2094-2099.

(11) Dotson, C. D.; Zhang, L.; Xu, H.; Shin, Y. K.; Vigues, S.; Ott, S. H.; Elson, A. E.; Choi, H. J.; Shaw, H.; Egan, J. M.; Mitchell, B. D.; Li, X.; Steinle, N. I.; Munger, S. D. Bitter taste receptors influence glucose homeostasis. PLoS One 2008, 3 (12), e3974.

(12) Jang, H. J.; Kokrashvili, Z.; Theodorakis, M. J.; Carlson, O. D.; Kim, B. J.; Zhou, J.; Kim, H. H.; Xu, X.; Chan, S. L.; Juhaszova, M.; Bernier, M.; Mosinger, B.; Margolskee, R. F.; Egan, J. M. Gut-expressed gustducin and taste receptors regulate secretion of glucagon-like peptide-1. Proc. Natl. Acad. Sci. U.S.A. 2007, 104, 15069-15074.

(13) Kim, U. K.; Jorgenson, E.; Coon, H.; Leppert, M.; Risch, N.; Drayna, D. Positional cloning of the human quantitative trait locus underlying taste sensitivity to phenylthiocarbamide. Science 2003, 299, $1221-1225$.

(14) Bufe, B.; Breslin, P. A. S.; Kuhn, C.; Reed, D. R.; Tharp, C. D.; Jay, P.; Slack, J. P.; Kim, U. K.; Drayna, D.; Meyerhof, W. The Molecular Basis of Individual Differences in Phenylthiocarbamide and Propylthiouracil Bitterness Perception. Curr. Biol. 2005, 15, 322-327.

(15) Floriano, W. B.; Hall, S.; Vaidehi, N.; Kim, U.; Drayna, D.; Goddard, W. A., III Modeling the human PTC bitter-taste receptor interactions with bitter tastants. J. Mol. Model. 2006, 12, 931-941.

(16) Miguet, L.; Zhang, Z.; Grigorov, M. G. Computational studies of ligand receptor interactions in bitter taste receptors. J. Recept. Signal Transductions Res. 2006, 26, 611-630.

(17) Biarnés, X.; Marchiori, A.; Giorgetti, A.; Lanzara, C.; Gasparini, P.; Carloni, P.; Born, S.; Brockhoff, A.; Behrens, M.; Meyerhof, W. Insights into the Binding of Phenyltiocarbamide (PTC) Agonist to Its Target Human TAS2R38 Bitter Receptor. PLoS One 2010, 5, e12394.

(18) Goddard, W. A., III; Kim, S. K.; Li, Y. Y.; Trzaskowski, B.; Griffith, A. R.; Abrol, R. Predicted 3D structures for adenosine receptors bound to ligands: Comparison to the crystal structure. J. Struct. Biol. 2010, 170, $10-20$.

(19) Lim, K. T.; Brunett, S.; Iotov, M.; McClurg, R. B.; Vaidehi, N.; Dasgupta, S.; Taylor, S.; Goddard, W. A., III Molecular dynamics for very large systems on massively parallel computers: The MPSim program. J. Comput. Chem. 1997, 18, 501-521. 
(20) Kam, V. W. T.; Goddard, W. A., III Flat-bottom strategy for improved accuracy in protein side-chain placements. J. Chem. Theory Comput. 2008, 4, 2160-2169.

(21) Kuntz, I. D.; Blaney, J. M.; Oatley, S. J.; Langridge, R.; Ferrin, T. E. A geometric approach to macromolecule-ligand interactions. J. Mol. Biol. 1982, 161, 269-288.

(22) Mayo, S. L.; Olafson, B. D.; Goddard, W. A., III DREIDING: a generic force field for molecular simulations. J. Phys. Chem. 1990, 94, 8897-8909.

(23) Bray, J. K.; Goddard, W. A., III The structure of human serotonin $2 \mathrm{c} \mathrm{G}$ protein-coupled receptor bound to agonists and antagonists. J. Mol. Graphics Modell. 2008, 27, 66-81.

(24) Spijker, P.; Vaidehi, N.; Freddolino, P.; Hilbers, P.; Goddard, W. A., III Dynamic behavior of fully solvated $\beta 2$-adrenergic receptor, embedded in the membrane with bound agonist or antagonist. Proc. Natl. Acad. Sci. U.S.A. 2006, 103, 4882-4887.

(25) Farrens, D.; Altenbach, C.; Yang, K.; Hubbell, W.; Khorana, H. Requirement of rigid-body motion of transmembrane helices for light activation of rhodopsin. Science 1996, 274, 768-770.

(26) Sheikh, S.; Zvyaga, T.; Lichtarge, O.; Sakma, T.; Bourne, H. Rhodopsin activation blocked by metal-ion-binding sites linking transmembrane helices C and F. Nature 1996, 383, 347-350. 